\title{
Análise estatística aplicada à gestão do tráfego em interseção semafórica
}

\author{
Statistical analysis applied to traffic management at signalized intersections \\ Análisis estadístico aplicado a la gestión del tránsito en intersecciones semaforizadas
}

Recebido: 26/01/2022 | Revisado: 30/01/2022 | Aceito: 06/02/2022 | Publicado: 07/02/2022

\author{
Oisy Hernandez Menendez \\ ORCID: https://orcid.org/0000-0001-5554-6199 \\ Universidade Federal do Oeste da Bahia, Brasil \\ E-mail: oisy.menendez@ufob.edu.br \\ Natalia Assunção Brasil Silva \\ ORCID: https://orcid.org/0000-0002-4296-6407 \\ Universidade Federal de São João del-Rei, Brasil \\ E-mail: natalia.brasil@ufsj.edu.br \\ Heraldo Nunes Pitanga \\ ORCID: https://orcid.org/0000-0001-5244-1180 \\ Universidade Federal de Juiz de Fora, Brasil \\ E-mail: heraldopitanga@yahoo.com.br
}

\begin{abstract}
Resumo
As interseções com semáforos tendem a ser os locais mais problemáticos do sistema viário, pois apresentam elevados níveis de congestionamento, acidentes, poluição do ar e grandes perdas econômicas. Com isso, os métodos e modelos que fornecem conhecimento dos parâmetros de eficiência da operação tornam-se cada vez mais protagonistas na gestão do trânsito urbano. No entanto, a disponibilidade de diferentes métodos e modelos pode levar à divergência entre os parâmetros dentro da mesma interseção. Assim, este estudo tem como objetivo analisar como a aplicação de testes estatísticos permite identificar instrumentos relevantes para a gestão do tráfego. A metodologia adotada consiste na utilização de dois simuladores VISSIM e Simtraffic, além do método de Webster, para obtenção dos parâmetros de eficiência de operação, atraso médio por veículo e comprimento da fila de um cruzamento semáforo com três acessos. Para a análise dos parâmetros, levando em consideração os diferentes métodos e modelos, e os três semáforos, foram aplicados os testes estatísticos ANOVA e Mann-Whitney. Os resultados mostram a importância da aplicação desses testes para melhorar a gestão do tráfego urbano, uma vez que esses testes determinam se os parâmetros de eficiência operacional diferem de acordo com o cenário, métodos e softwares aplicados.
\end{abstract}

Palavras-chave: Interseção; Atraso médio; Comprimento da fila; Test Mann-Whitney; ANOVA.

\begin{abstract}
Traffic light intersections tend to be the most problematic places in the road systems, as they present high levels of congestion, accidents, air pollution and high economic losses. With this, the methods and models that provide knowledge of the efficiency parameters of the operation become more and more protagonists in the management of urban traffic. However, the availability of different methods and models can lead to divergence between the parameters within the same intersection. Thus, this study aims to analyze how the application of statistical tests allows the identification of relevant instruments for traffic management. The methodology consists of using two VISSIM and Simtraffic simulators, and the Webster method to obtain the parameters of operation efficiency, average delay and the queue length of the tail of a traffic light intersection with three accesses. For the analysis of the parameters, taking into account different methods and models, in three traffic light cycles, the statistical tests of ANOVA and Mann-Whitney were applied. The results show the importance of applying these tests to improve urban traffic management, since these tests determine whether the operational efficiency parameters differ according to the scenario, methods and software applied.
\end{abstract}

Keywords: Intersection; Delay; Queue length; Test Mann-Whitney; ANOVA.

\section{Resumen}

Las intersecciones semaforizadas tienden a ser los locales más problemáticos de los sistemas viales, por presentar altos niveles de congestionamiento, accidentes, contaminación del aire y elevadas pérdidas económicas. Con ello, los métodos y modelos que proporcionan el conocimiento de parámetros de eficiencia de la operación se vuelven cada vez más protagonistas en la gestión del tránsito urbano. Sin embargo, la disponibilidad de diferentes métodos y modelos puede producir divergencia entre los parámetros dentro de la misma intersección. Por tanto, este estudio tiene como objetivo analizar como la aplicación de pruebas estadísticas permite identificar instrumentos que son relevantes para la gestión del tránsito. La metodología adoptada consiste en utilizar dos simuladores VISSIM y Simtraffic, y el método de Webster para obtener los parámetros de eficiencia de la operación, demora promedio por vehículo y la 
longitud de la cola de una intersección semaforizada con tres accesos. Para el análisis de los parámetros, teniendo en cuenta los diferentes métodos y modelos, y los tres ciclos semafóricos, se aplicaron las pruebas estadísticas de ANOVA y Mann-Whitney. Con los resultados se observa la importancia de aplicar estas pruebas para la mejora de la gestión del tránsito urbano, dado que dichas pruebas determinan si los parámetros de eficiencia operacional difieren según el escenario, métodos y software aplicado.

Palabras clave: Intersección; Demora; Longitud de la cola; Test Mann-Whitney; ANOVA.

\section{Introdução}

O semáforo é uma das ferramentas mais eficientes para controlar o tráfego, pois evitam a colisão de veículos em movimento nas interseções (Oskerbski et al., 2016). Além disso, conforme ressaltado por Elefteriadou (2014), normalmente, o ciclo semafórico é determinado a fim de maximizar a capacidade da via que reflete no atraso dos veículos, na eficiência da operação de tráfego e na segurança da rede de tráfego (Ma et al., 2020).

Nas interseções viárias com controle semafórico são altos os índices de congestionamento, acidentes, poluição do ar e perdas econômicas (Jíménez et al., 2015; Ma et al., 2020; Otković et al., 2020). Por isso, no âmbito técnico-científico da engenharia de tráfego, são recorrentes, estudos que visam identificar fatores que influenciam na eficiência do controle semafórico nas interseções. Dentre eles, cita-se Khosla \& Williams (2006) que avaliaram o efeito do tempo verde na descarga da fila de veículos e Torquato et al. (2020) que analisaram o efeito do tempo perdido no momento da partida do veículo e do fluxo de saturação. Os fatores largura da faixa, inclinação, presença de estacionamentos e paradas de ônibus e movimentos de conversão, também são considerados pelo modelo HCM (TRB, 2010). Outras pesquisas para avaliar a capacidade da interseção semaforizada recorreram aos parâmetros de desempenho do tráfego, como a média do atraso veicular e o comprimento de fila dos veículos (Osorio et al., 2015; Mahmud et al., 2019; Datta et al., 2020; Wang et al., 2020).

Os parâmetros, atraso e comprimento da fila em interseções semaforizadas podem ser determinados a partir da aplicação de métodos convencionais, HCM (TRB, 2010) e Webster (1964), ou pelo uso de simuladores de tráfego (De Oliveira, 2016; Saha et al., 2016; Shaaban \& Kim, 2015; Sun et al., 2013). No entanto, destaca-se que os métodos convencionais tendem a superestimar os atrasos, pois suas formulações foram desenvolvidas para países com tráfego homogêneo e motoristas disciplinados (Cal et al., 2010; Ma et al., 2020; Otković et al., 2020; Saha et al., 2016). Enquanto, os simuladores de tráfego possibilitam, a partir da modelagem do tráfego urbano, a avaliação do desempenho das interseções com controle semafórico e a proposição de estratégias que otimizam o controle do tráfego nas mesmas, além de ser uma tecnologia de pesquisa econômica e confiável que representa a formação e a descarga das filas de veículos nas interseções (Araújo \& Castro, 2018; Datta et al., 2020; Mahmud et al., 2019; Tianzi et al., 2013). Portanto, é necessário investigar o desempenho destes modelos para condições de tráfego heterogêneo antes de sua efetiva implementação em campo.

Devido à disponibilidade de diferentes softwares de simulação do tráfego e à importância dos parâmetros de desempenho da rede viária no planejamento urbano (Datta et al., 2020; Otković et al., 2020; Saha et al., 2016), estudos comparativos entre parâmetros de desempenho de trafego em interseções semafóricas podem auxiliar na análise comparativa de modelos de simulação de tráfego apresentados por diferentes softwares e destes com os parâmetros de desempenho obtidos aplicando os métodos manuais.

Logo, o objetivo desta pesquisa foi analisar como a aplicação de testes estatísticos podem auxiliar no planejamento do tráfego, tendo em vista que a aplicação de diferentes métodos e modelos podem apresentar parâmetros de desempenho divergentes para uma mesma interseção viária. Para tal, valeu-se de testes estatísticos na análise de parâmetros de desempenho de operacional, atraso médio por veículo e comprimento da fila de veículos, determinados por dois softwares de microssimulação e o método manual de Webster (1964), para diferentes aproximações semafóricas. 


\subsection{Referencial Teórico}

\subsubsection{Métodos convencionais para a determinação de parâmetros de desempenho das interseções}

Dentre os diversos métodos aplicados para a determinação dos parâmetros que caracterizam as interseções semafóricas, a saber, duração do ciclo semafórico, atraso veicular e comprimento da fila, os amplamente aplicados são o modelo HCM (TRB, 2010) e o modelo determinístico de Webster (1964) baseado na teoria de filas, ambos métodos desenvolvidos para tráfego homogêneo com atrasos veiculares mínimos (Cal et al., 2010; Saha et al., 2016).

Nesse sentido, Dion et al. (2004) ao compararem o atraso veicular na interseção semaforizada em condições de tráfego sub-saturado e altamente saturado, utilizando modelos de atraso determinísticos e estocásticos, concluíram que nas condições de baixa demanda de tráfego, todos os modelos apresentaram resultados semelhantes. No entanto, Dion et al. (2004) verificaram que quando a relação volume e capacidade se aproxima de 0,7 , as discrepâncias entre os valores de atraso estimados pela aplicação dos métodos convencionais e aqueles observados em campo começaram a aumentar. Chaudhry e Ranjitkar (2013) desenvolveram um modelo de atraso baseado em dados coletados em interseções semaforizadas e comprovaram que o modelo de Webster (1964) superestima o atraso. Tendo em vista tais observações, outros estudos, como o de Raval e Gundaliya (2012) propuseram uma modificação na fórmula de atraso do método Webster (1964), acrescentando um fator de ajuste que considera o atraso observado em campo e os atrasos obtidos, assim, ao aplicar o fator de ajuste os atrasos obtidos foram próximos aos dados de campo.

Em relação aos estudos com aplicação do modelo HCM (TRB, 2010), Kumar e Dhinakaran (2013) mediram o atraso em campo e compararam com o atraso obtido a partir da aplicação do modelo de HCM 2010, e observaram que o atraso em campo difere do obtido pelo modelo do HCM. Saha et al. (2016) compararam os atrasos em campo com os obtidos ao aplicar os modelos do HCM (2010) e Webster (1964) além de utilizar métodos probabilísticos de regressão e o modelo de expansão de Taylor. E neste estudo, Saha et al. (2016) verificou que o atraso medido diretamente em campo, com base no comprimento da fila e a regra de Simpson foi mais preciso do que o atraso calculado pelos métodos convencionais.

As diferenças dos atrasos veiculares e comprimento da fila determinados entre os métodos convencionais e aqueles observados em campo, segundo (Chaudhry, 2015), geralmente está baseado no fato dos países em desenvolvimento apresentarem trafego altamente heterogêneo e grau de saturação maior 0,60.

\subsubsection{Simuladores de tráfego}

Diante das complexidades enfrentadas pelos planejadores de tráfego em obter através da aplicação dos modelos convencionais parâmetros condizentes com o tráfego em campo, destaca-se os simuladores de tráfego. Os simuladores de tráfego são ferramentas de abordagem objetiva e flexível na avaliação de alternativas de projeto e gerenciamento do tráfego, que permitem replicar a dinâmica real da rede de tráfego e estimar os impactos na qualidade do ar, taxas de consumo de combustível e fatores de risco de acidentes (Mahmud et al., 2019). Contudo, Tianzi et al. (2013) destacam que os valores dos parâmetros de desempenho de uma interseção viária fornecidos pelos simuladores variam conforme o software de simulação do tráfego.

De acordo com o Planing Transport Verkehr (PTV AG Corporation, 2016), o software VISSIM é um programa de microssimulação para modelagem multimodal de operações de transportes. Este software é um modelo de simulação de tráfego microscópico estocástico, capaz de representar o comportamento do tráfego em vias urbanas (Park \& Qi, 2005; Obelheiro et al., 2010). Segundo Mahmud et al. (2019), dentre as principais vantagens do VISSIM citam-se as possibilidades de analisar uma ampla gama de atividades de tráfego, possuir um sistema de roteamento dinâmico, adicionar o objeto no ponto exato desejado na rede de tráfego e com o efeito desejado nos usuários da estrada, além do usuário escolher a duração da análise.

Ayala (2013) destaca como parâmetros de simulação no VISSIM a distância de mudança de faixa, distância de parada, 
distância de look-head, distância média de parada, distância mínima de observação, distância mínima lateral, distribuição de velocidade desejada, fluxo de saturação, distância de segurança desejada, número de veículos precedentes observados, tempo de headway, mínimo headway, distância de parada de emergência e velocidade desejada. Mahmud et al. (2019) acrescentam, como outros parâmetros simulados pelo VISSIM, o comportamento do condutor em termos de aceleração, desaceleração, configurações de faixa, taxa de frenagem, aceitação de folga e rejeição e o nível de serviço. Os dados de saída do VISSIM são uma animação gráfica contínua, que representa o tráfego na rede viária, a partir de dados estatísticos de volume, velocidade, tempo de viagens, atrasos e paradas e dados coletados em pontos determinados pelo usuário através de ferramentas específicas (Obelheiro et al., 2010).

Embora o software VISSIM seja amplamente utilizado para avaliar as condições de circulação do tráfego em interseções, existem outros programas como o Synchro que realiza uma simulação macroscópica com modelo de análise determinístico (Teixeira, 2008; Chaudhry, 2015), e SimTraffic que realiza a microssimulação da rede modelada no Synchro, ambos avaliam a capacidade de uma interseção baseada nas especificações do Highway Capacity Manual (HCM, 2010).

O software Synchro tem uma interface gráfica que reduz o tempo de entrada e elimina a necessidade de manter vários arquivos durante a análise, oferecendo a capacidade de importar e exportar dados entre muitos programas diferentes de software de tráfego. Esse software gera relatórios e o usuário pode definir os níveis de rede, arterial ou de interseção, limitar as medidas de eficácia ao nível da faixa, além de ter a capacidade de designar e manipular zonas ou partições (ATAC, 2003; Koonce et at., 2008). O Synchro tem como principais vantagens permitir a otimização dos ciclos e das fases semafóricas sem ter que modelar outras situações, a sincronização de vários semáforos em redes, sendo totalmente interativo, e a atualização dos cálculos para cada modificação realizada (Trafficware, 2017).

No software Synchro, tem-se o pacote SimTraffic que realiza uma modelação microscópica de uma variedade de controles de tráfego, incluindo uma rede com sinalização semafórica operando em diferentes comprimentos de ciclo ou operando sobre condições totalmente ativadas pelo tráfego. A diferença entre o SimTraffic e o VISSIM está no modelo de seguimento de carro (car-following), em que o carro de reboque segue o carro da frente com 1 segundo de avanço entre os veículos (Shaaban \& Kim, 2015; Trafficware, 2017).

Além destes, podem ser citados outros softwares simuladores de tráfego empregados em pesquisas, como CORSIM (Bloomberg \& Dale, 2000; Yizheng et al., 2012; Sun et al., 2013), SIDRA (Lee, 2010; Tianzi et al., 2013), Rodel (Lee, 2010; Shaaban \& Kim, 2015), PARAMICS (Steven L. Jones et al., 2004; Mahmud et al., 2019) e AIMSUN (Ayala, 2013; Steven L. Jones et al., 2004; Mahmud et al., 2019).

São vários os estudos que comparam os softwares simuladores a partir dos parâmetros de desempenho do tráfego. Bloomberg e Dale (2000) compararam os softwares VISSIM e CORSIM, a partir dos aspectos referentes ao processo de codificação da rede viária, tempo de ciclo semafórico, recursos de animação e dados de saída. Yizheng et al. (2012) valeram-se do método de análise de sensibilidade para comparar os respectivos desempenhos do VISSIM e do CORSIM, utilizando como principais indicadores a simplicidade de operação, o atraso médio dos veículos e o volume de tráfego na seção de controle viário.

Tianzi et al. (2013) concentraram suas pesquisas nos softwares VISSIM e SIDRA, sob as perspectivas da simplicidade da operação e do erro nos dados de saída. De acordo com essas pesquisas, a comparação entre os simuladores é baseada nos dados de entrada para simulação, nos parâmetros de desempenho viário e nos dados de saída fornecidos pelos softwares de simulação.

Lee (2010), ao analisar uma rotatória utilizando três softwares de simulação, a saber, VISSIM, Rodel e SIDRA, concluiu que os três superestimam a capacidade. O SIDRA e o VISSIM superestimam também os atrasos e comprimentos de fila. Além disso, verificou-se que a confiabilidade do simulador de tráfego está intrinsicamente relacionada à sua capacidade de 
reproduzir dentro de uma margem aceitável de erro.

Sun et al. (2013) compararam os modelos dos softwares VISSIM e CORSIM com base em quatro índices principais: usabilidade do software, atraso médio de controle, duração média da fila e volume de tráfego transversal, concluindo que ambos os softwares conseguem uma simulação do volume de tráfego bem próxima dos dados de campo e que, com o VISSIM, é possível a obtenção de atrasos médios e comprimentos de fila mais próximo da situação real.

Mahmud et al. (2019) compararam os microssimuladores VISSIM, PARAMICS e AIMSUN e apontaram que a maior parte do desenvolvimento e aplicação do modelo de simulação é para ambientes de tráfego homogêneo, apresentando limitações ao analisar o comportamento dinâmico do tráfego e na avaliação de conflitos.

Shaaban \& Kim (2015) compararam o atraso médio obtido a partir de simulações realizadas no VISSIM e no Simtraffic em uma rotatória, concluindo que, para volumes de tráfego menores, não existe diferença significativa entre os atrasos, mas quando o volume é elevado, o VISSIM apresenta atrasos médios mais altos do que os do SimTraffic. Chaudhry (2015) utilizou também os microssimuladores VISSIM e SimTraffic em uma interseção sinalizada e observou que, com o VISSIM, são obtidas capacidades da via maiores que com o SimTraffic, mas os comprimentos de fila e atrasos são inferiores.

\section{Metodologia}

\subsection{Local de estudo}

O local de estudo da pesquisa (Figura 1) é uma interseção semafórica entre a Avenida Severino Vieira e a Rua Floriano Peixoto, situada no município de Barreiras, considerado o mais populoso da região ocidental do estado da Bahia. De acordo com o Instituto Brasileiro de Geografia e Estatística (IBGE) a população de Barreiras em 2021 foi estimada em 158.432 habitantes. A escolha dessa interseção semafórica, formada por três aproximações, foi motivada pela concentração de acidentes no local, aumento da frota de veículos no munícipio e por promover o acesso às principais rodovias do canal MATOPIBA interligando os sistemas viários entre as regiões Leste e Oeste do município de Barreiras (DNIT, 2006).(Manual de Estudos de Tráfego, 2006)

Figura 1. Interseção semaforizada entre a Avenida Severino Vieira e a Rua Floriano Peixoto.

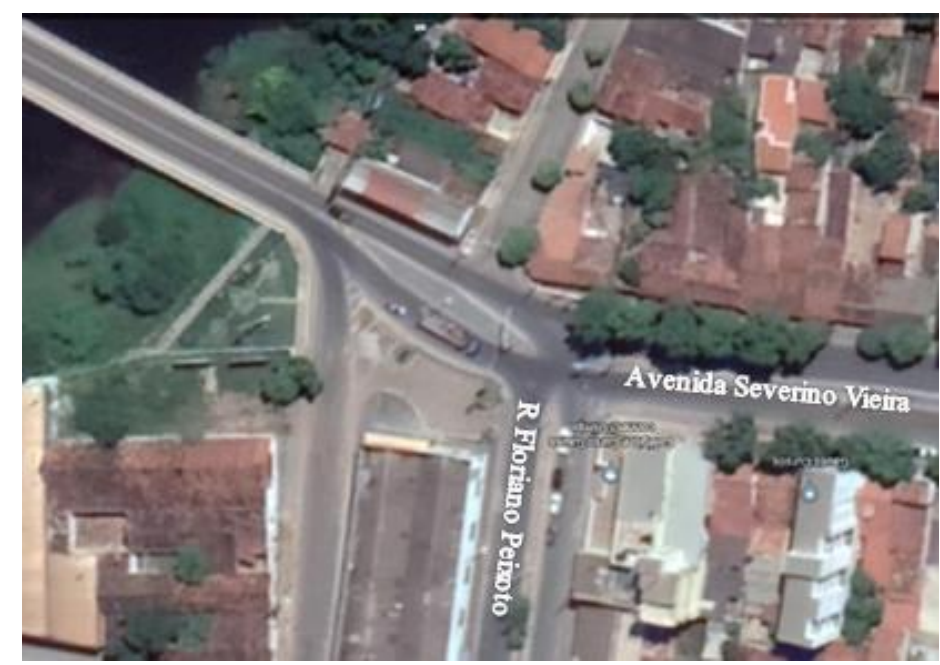

Fonte: Google Earth (2021).

\subsection{Modelagem do tráfego na interseção semaforizada}

Para a modelagem da interseção nos softwares SimTraffic $®$ [da versão 10.1 do Synchro Studio da Trafficware LLC (2016)] e VISSIM® [versão 9.00-09 da PTV AG Corporation (2016)], foi necessário determinar as características geométricas 
das vias, do tráfego e do sistema de controle das aproximações que formam a interseção viária, de acordo com a Tabela 1.

Tabela 1. Características necessárias para a modelagem da interseção no software de microssimulação de tráfego.

\begin{tabular}{|c|c|c|}
\hline $\begin{array}{l}\text { Característi } \\
\text { cas }\end{array}$ & Informações & Metodologia \\
\hline \multirow{4}{*}{$\begin{array}{l}\text { Geometria das vias } \\
\text { de cada } \\
\text { aproximação }\end{array}$} & Inclinação das vias & \multirow{4}{*}{ Visita in loco } \\
\hline & Velocidade diretriz das vias & \\
\hline & Largura das faixas de trânsito & \\
\hline & Número de faixas de trânsito por aproximação & \\
\hline \multirow{4}{*}{ Tráfego } & Volume de tráfego no horário de máxima demanda - Hora Pico (HP) & \multirow{4}{*}{$\begin{array}{l}\text { Videoteipe, classificatório e } \\
\text { direcional }^{(1)}(\mathrm{DNIT}, 2006)\end{array}$} \\
\hline & Fator Hora Pico (FHP) & \\
\hline & Porcentagem de veículos pesados (DNIT, 2006) & \\
\hline & Movimentos por aproximação & \\
\hline \multirow{2}{*}{$\begin{array}{l}\text { Sistema de } \\
\text { controle de tráfego }\end{array}$} & Ciclo semafórico & \multirow{2}{*}{ Visita in loco } \\
\hline & Fases do ciclo semafórico & \\
\hline
\end{tabular}

Fonte: Autores (2021).

Como os modelos de microssimulação de tráfego gerados pelos softwares são estocásticos, foram realizadas, como parte do processo de modelagem, as etapas de calibração e validação dos mesmos. Para a calibração dos modelos de microssimulação, definiu-se o volume de tráfego por hora nas entradas das aproximações da interseção viária como medida de desempenho operacional. Assim, de acordo com Shaaban \& Radwan (2005), inicialmente, foram realizadas várias simulações para verificar se os resultados médios da medida de desempenho refletiam a condição de tráfego em campo. Após essa verificação, foi determinado o número mínimo de simulações necessárias para cada modelo, de acordo com a média e o desvio padrão da medida de desempenho.

Para a determinação do número mínimo de simulações (N), aplicou-se a equação 1 (Shaaban \& Radwan, 2005) e a equação 2 (Dowling et al., 2004). O valor $\mathrm{N}$ definido para os modelos de microssimulação de cada software foi o maior dentre os valores determinados pela aplicação das equações 1 e 2. Para a aplicação destas equações, realizaram-se vinte simulações de tráfego para as estimativas iniciais das médias $(\mu)$ e desvios padrão (s) dos valores do volume de tráfego de entrada nas aproximações, sendo $\mathrm{t} \_(\propto / 2)$ e t_((1- $\left.\left.\propto\right), \mathrm{N}-1\right)$ o valor crítico da distribuição-t para um intervalo de confiança (IC) igual a 95\%, nível de significância $(\alpha)$ de $5 \%$ e erro admissível $(\varepsilon)$ igual a $5 \%$.

$$
\begin{aligned}
\mathrm{N} & =\left(\mathrm{t}_{\propto / 2} \cdot \frac{\mathrm{s}}{\mu \varepsilon}\right)^{2} \\
C I_{1-\propto \%} & =2 x t_{(1-\propto), N-1} \frac{s}{\sqrt{N}}
\end{aligned}
$$

A próxima etapa no processo de calibração foi a minimização da diferença entre os valores do volume de tráfego nas aproximações obtidos em campo e os valores simulados. Nesse caso, valeu-se do cálculo do GEH (Geoffrey E. Havers), baseado no teste estatístico do chi-quadrado $\left(\chi^{2}\right)$, conforme apresentado na equação 3 , em que $\mathrm{E}$ é o volume de tráfego estimado pelo modelo de microssimulação e V é o volume de tráfego em campo (Dowling et al., 2004).

$$
G E H=\sqrt{\frac{2(E-V)^{2}}{E+V}}
$$

O modelo de microssimulação foi considerado calibrado quando o valor do GEH em cada aproximação foi inferior a 5 (GEH < 5) em pelo menos 85\% das N simulações (Dowling et al., 2004; Nyame-Baafi et al., 2018). Após a calibração dos modelos de microssimulação, realizou-se a validação visual do modelo, através da verificação das velocidades em alguns 
trechos das vias e dos conflitos entre veículos (conforme movimentos permitidos) e número de veículos na fila.

\subsection{Parâmetros de análise da interseção semaforizada}

Com os modelos de microssimulação calibrados e validados, avaliaram-se os parâmetros de desempenho operacional nas faixas de tráfego das aproximações na interseção semafórica pelo método de Webster (1964), aplicando a metodologia HCM (2010), conforme as equações apresentadas na Tabela 2.

Tabela 2. Parâmetros de desempenho operacional das aproximações na interseção semafórica.

\begin{tabular}{|c|c|c|}
\hline Parâmetros & Equações & \\
\hline $\begin{array}{l}\text { Atraso médio por veículo } \\
\text { (Webster, 1964) }\end{array}$ & $\begin{array}{l}d=\frac{c \cdot(1-\lambda)^{2}}{2 \cdot(1-\lambda x)}+\frac{x^{2}}{2 \cdot(1-x)}-0,65 \cdot\left(\frac{c}{q^{2}}\right)^{1 / 3} \cdot x^{(2+5 \lambda)} \\
\text { em que: } \\
\text { d: atraso médio (segundos); } \\
\text { c: ciclo (segundos); } \\
\lambda: \text { relação verde efetivo / tempo de ciclo; } \\
\text { q: demanda (veículos/segundos); } \\
\text { x: grau de saturação }\end{array}$ & (4) \\
\hline Fila máxima & $\begin{array}{l}f_{\max }=F \cdot \frac{\left(t_{c}-t_{v} \text { ef etivo }\right)}{3600} \\
\text { em que: } \\
\mathrm{f}_{\max }: \text { fila máxima (ucp); } \\
\mathrm{F}: \text { taxa de fluxo (ucp/h); } \\
\mathrm{t}_{\mathrm{c}} \text { : tempo de ciclo (segundos); } \\
\mathrm{t}_{\mathrm{v}} \text { efetivo: tempo de verde efetivo (segundos) }\end{array}$ & $(5)$ \\
\hline Capacidade & $\begin{array}{l}C_{i}=S_{i} \cdot\left(\frac{g_{i}}{c}\right) \\
\text { em que: } \\
\text { Ci: capacidade (unidade); } \\
\mathrm{S}_{\mathrm{i}}: \text { fluxo de saturação }(\mathrm{ucp} / \mathrm{h}) \\
\mathrm{g}_{\mathrm{i}}: \text { verde efetivo (segundos); } \\
\text { C: ciclo semáforico (segundos), }\end{array}$ & (6) \\
\hline Razão v/c & v/c: razão entre o volume máximo e a capacidade da faixa estudada & $(7)$ \\
\hline
\end{tabular}

Fonte: Autores (2021).

\subsection{Análises estatísticas}

Inicialmente, com a utilização do software IBM SPSS, versão 20.0, realizou-se o teste de normalidade Shapiro-Wilk (S-W), intervalo de confiança de 95\% [nível de significância $(\alpha)$ de 5\%], com os valores dos parâmetros de desempenho operacional, "atraso médio por veículo" e "comprimento da fila de veículos", nas faixas de tráfego das aproximações na interseção semafórica, obtidos pelos modelos de microssimulação dos softwares SimTraffic $®$ e VISSIM $®$. Este teste forneceu o parâmetro valor-p ou significância, sabendo que H0 corresponde à distribuição normal dos parâmetros determinados nas $\mathrm{N}$ simulações. Para valor-p $\leq 0,05$, rejeita-se $\mathrm{H} 0$, e os dados são classificados como não-paramétricos; para valor- $\mathrm{p}>0,05$, não se rejeita H0, ou seja, a distribuição é normal.

Os parâmetros de desempenho operacional supracitados foram comparados estatisticamente (Bloomberg \& Dale, 2000; Shaaban \& Kim, 2015). Para tal comparação, valeu-se da aplicação dos métodos ANOVA (Shaaban \& Kim, 2015; Wang et al., 2018), para os dados paramétricos, e Mann-Whitney (Ayala, 2013), para os dados não paramétricos.

\section{Resultados e Discussão}

\subsection{Caracterização geral e análise descritiva}

Na Tabela 3 são apresentadas as características das aproximações semafóricas da interseção viária necessárias para a 
sua modelagem nos softwares de microssimulação de tráfego.

Tabela 3. Características das aproximações semafóricas da interseção viária.

\begin{tabular}{|c|c|c|c|c|c|c|}
\hline Características & \multicolumn{2}{|c|}{$\begin{array}{l}\text { Aproximação Leste } \\
\text { (AL) }\end{array}$} & \multicolumn{2}{|c|}{$\begin{array}{l}\text { Aproximação Oeste } \\
\text { (AO) }\end{array}$} & \multicolumn{2}{|c|}{$\begin{array}{l}\text { Aproximação Norte } \\
\text { (AN) }\end{array}$} \\
\hline Nomenclatura & \multicolumn{4}{|c|}{ Avenida Severino Vieira - BR 242} & \multicolumn{2}{|c|}{ Rua Floriano Peixoto } \\
\hline Número de faixas de tráfego & \multicolumn{2}{|c|}{02} & \multicolumn{2}{|l|}{02} & \multicolumn{2}{|c|}{02} \\
\hline \multicolumn{7}{|l|}{ Movimentos por aproximação } \\
\hline Largura das faixas de tráfego (metro) & \multicolumn{2}{|c|}{3,30} & \multicolumn{2}{|c|}{3,60} & \multicolumn{2}{|c|}{3,40} \\
\hline Inclinação da via & \multicolumn{2}{|c|}{ Ascendente } & \multicolumn{2}{|c|}{ Descendente } & \multicolumn{2}{|c|}{ Descendente } \\
\hline Presença da faixa de conversão & \multicolumn{2}{|c|}{ Direita } & \multicolumn{2}{|c|}{ Esquerda } & \multicolumn{2}{|c|}{ Direita e Esquerda } \\
\hline Faixa de tráfego & AL1 & AL2 & $\mathrm{AO} 1$ & $\mathrm{AO} 2$ & AN1 & AN2 \\
\hline $\begin{array}{l}\text { Movimento permitido nas faixas de tráfego } \\
\text { (Erro! Fonte de referência não encontrada.) }\end{array}$ & $01-02$ & 03 & 04 & $05-06$ & $07-08$ & 09 \\
\hline $\begin{array}{l}\text { Volume de tráfego no horário de máxima } \\
\text { demanda - (ucp) }\end{array}$ & 253 & 427 & 694 & 278 & 167 & 298 \\
\hline Fator hora pico & 0,87 & 0,85 & 0,82 & 0,87 & 0,83 & 0,93 \\
\hline Veículo pesado (\%) & 8 & 6 & 7 & 9 & 7 & 4 \\
\hline
\end{tabular}

Fonte: Autores (2021).

Na Figura 2 são apresentadas as aproximações semafóricas da interseção viária, assim como os movimentos permitidos nas faixas de tráfego, conforme foi descrito na Tabela 3.

Figura 2. Interseção Avenida Severino Vieira e Rua Floriano Peixoto.

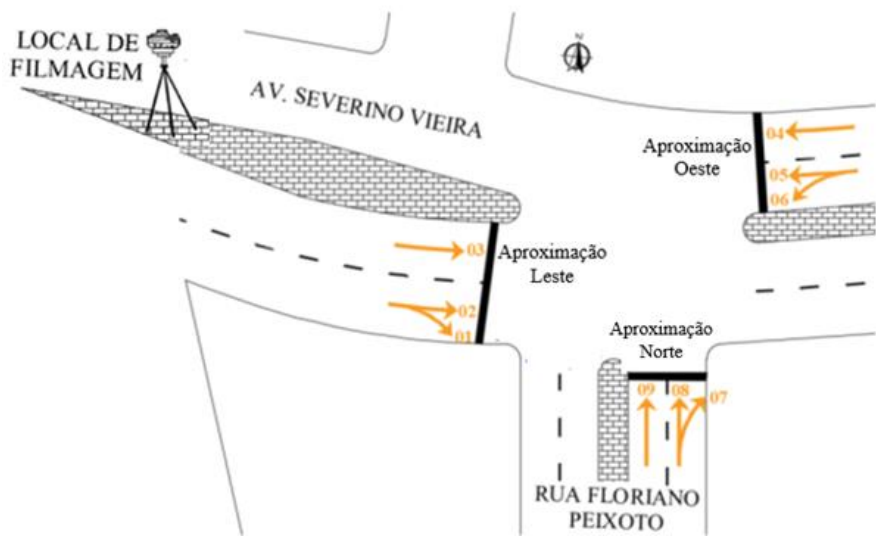

Fonte: Autores (2021).

Observou-se que os maiores volumes de tráfego na interseção viária são provenientes das aproximações oeste e leste, respectivamente. Conforme observado em campo, o controle semafórico nas aproximações da interseção viária apresentou três ciclos semafóricos. Neste estudo, cada ciclo semafórico (80, 95 e 100 segundos) correspondeu a um cenário de simulação [cenário $1(\mathrm{C} 1)$, cenário $2(\mathrm{C} 2)$ e cenário $3(\mathrm{C} 3)$, respectivamente, sendo o cenário 2 o ciclo ótimo calculado pelo método de Webster (1964)].

Na Figura 3 são apresentados os diagramas de distribuição dos programas semafóricos dos três cenários. 
Figura 3. Diagramas de distribuição dos C1, C2 e C3 e plano de fases dos programas semafóricos.

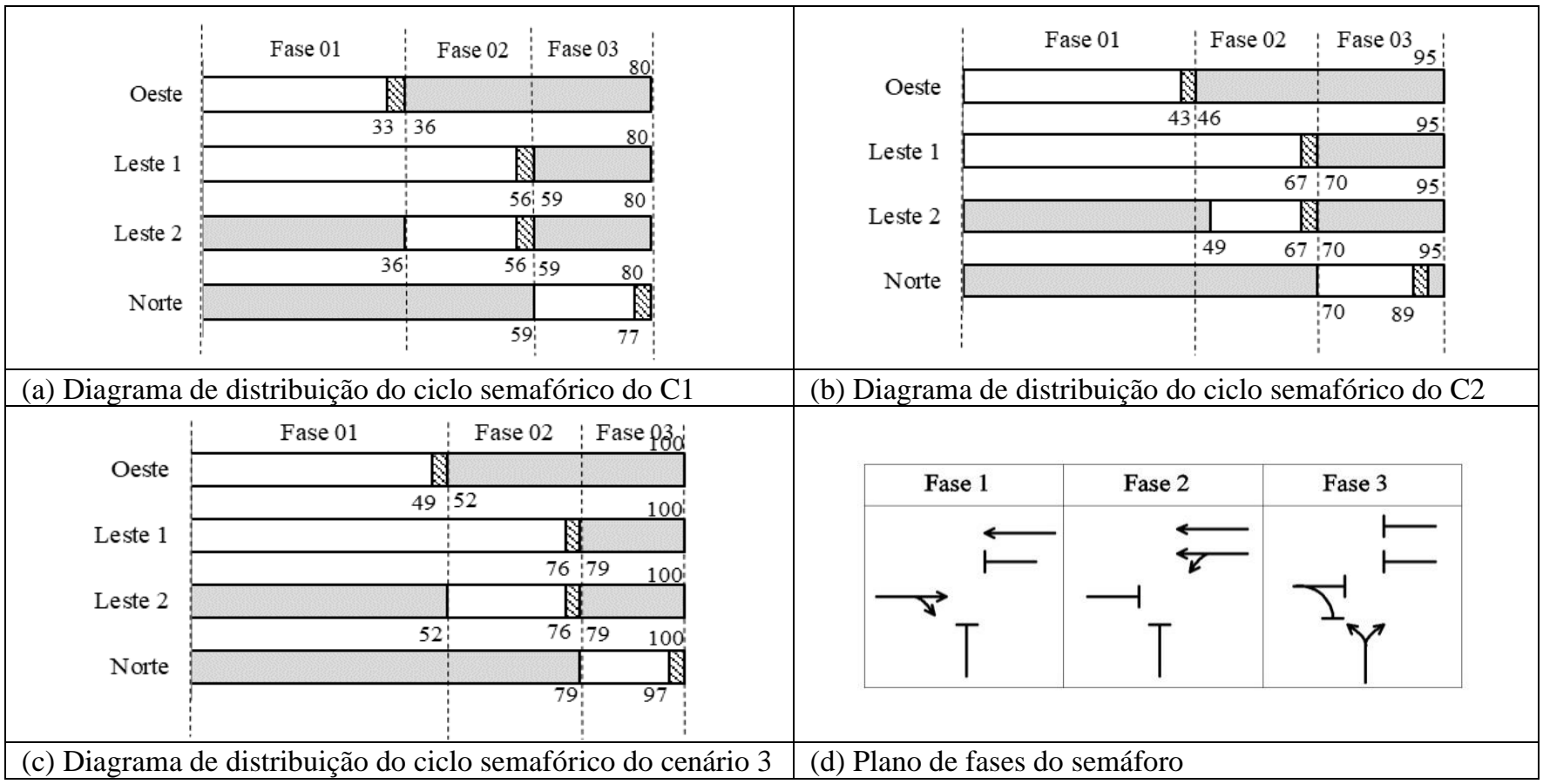

Fonte: Autores (2021).

O plano de fases é o mesmo para todos os cenários. A fase 1 permite os movimentos $1,2,3$ e 4 , a fase 2 permite os movimentos 4,5 e 6 , e a fase 3 permite os movimentos 7,8 e 9 .

\subsection{Calibração da microssimulação}

Após a inserção dos dados de entrada nos softwares SimTraffic $®$ e VISSIM®, determinou-se, com a aplicação dos métodos Shaaban (2005) e Dowling (2004), a partir dos volumes de tráfego provenientes das vinte simulações dos modelos iniciais e de parâmetros estatísticos, que os números mínimos de simulações dos modelos para cada software são 23 para o SimTraffic ${ }^{\circledR}$ e 29 para o VISSIM®. Assim, ao considerar o maior valor mínimo de simulações, foram realizadas 30 simulações dos modelos de microssimulação de tráfego em cada software.

A partir dos valores do volume de tráfego nas aproximações da interseção viária obtidos em campo no horário de máxima demanda e dos valores de volume de tráfego verificados nessas aproximações nas 30 simulações dos modelos de tráfego, foi possível determinar o GEH para os $\mathrm{C} 1, \mathrm{C} 2$ e C3. Observou-se que as porcentagens de simulações com valores de GEH inferiores a 5 foram superiores a $85 \%$, inferindo-se, assim, que os modelos de microssimulação em ambos os softwares e cenários simulados estavam calibrados (Dowling et al., 2004).

\subsection{Características operacionais da interseção}

Na Figura 4 são apresentadas as características operacionais das faixas de tráfego das aproximaçoes, capacidade, relação volume/capacidade e o nível de serviço das faixas de tráfego que formam a interseção viária para os cenários C1, C2 e C3. Os volumes de tráfego considerados foram os determinados pelos modelos de microssimulação e os observados em campo, a capacidade das faixas de tráfego foi determinada pelo método manual Webster (1964) (equação 6), e o nível de serviço, representado pelas letras entre parênteses, foram obtidos nos modelos de microssimulação e a partir das definições presentes no manual de estudos de tráfego (DNIT, 2006). 
Para a determinação da capacidade de tráfego nas aproximações cujas faixas de tráfego apresentam o mesmo controle semafórico, considerou-se o maior fluxo de saturação observado em campo entre essas faixas de tráfego, razão pela qual as capacidades de tráfego nas aproximações leste (AL1 e AL2) e norte (AN1 e AN2) foram iguais.

Figura 4. Características operacionais das faixas de tráfego das aproximações da interseção viária para C1, C2 e C3.

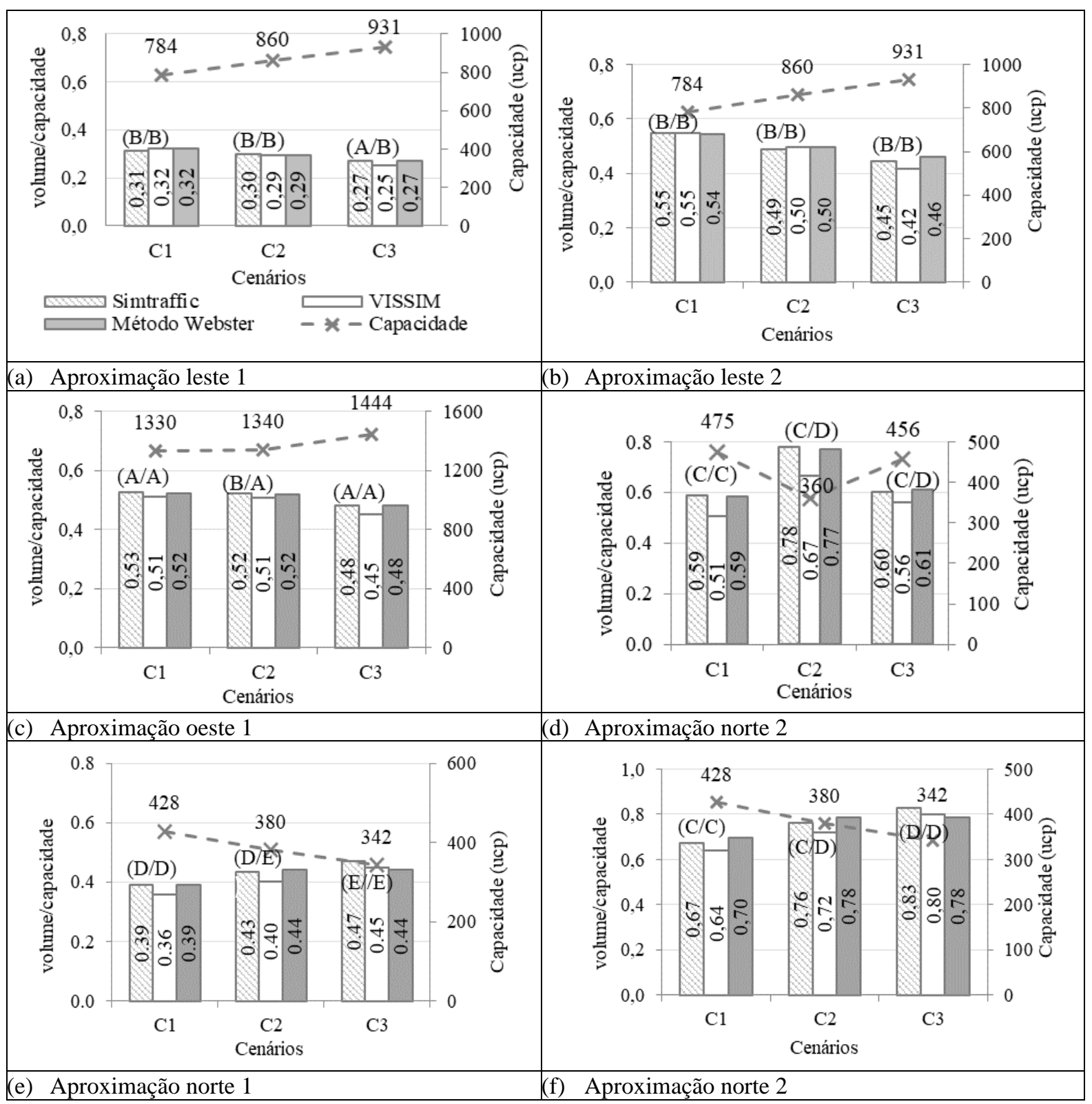

Fonte: Autores (2021).

Observou-se, entre os cenários analisados, que o aumento do ciclo semafórico proporcionou maior capacidade de tráfego nas aproximações AL1, AL2 e AO1, devido ao prolongamento do tempo de verde nessas faixas de tráfego. Entretanto, notou-se comportamento contrário nas aproximações AO2, AN1 e AN2, o que é condizente com as características operacionais das aproximações, tendo em vista que nesses locais, além do aumento do ciclo semafórico que proporciona uma redução do tempo de verde, existe uma união de duas vias (aproximações AN1 e AN2), e para AO2 poderia estar relacionado com a existência de dois movimentos em uma única faixa. Além disso, através da análise do nível de serviço das faixas de tráfego, 
verificou-se que no $\mathrm{C} 1$ os níveis de serviço das aproximações foram entre $\mathrm{A}$ e $\mathrm{D}$, e nos cenários 2 e 3 notou-se o nível serviço E na AN1, sendo inferior ao das demais aproximações.

Logo, infere-se que os aumentos do ciclo semafórico proporcionaram a melhora da capacidade de tráfego nas aproximações de maiores demandas. O cenário C1, cujo ciclo semafórico foi de 80 segundos, oferece as melhores condições operacionais (densidade de tráfego) para todas as aproximações da interseção.

Cabe ressaltar também que a relação entre o volume de tráfego (v) (seja aquele observado em campo ou determinado pelos modelos de microssimulação) e a capacidade (c) das faixas de tráfego foram inferiores a 1, isto é, a demanda de veículos foi inferior ao número máximo de veículos que as faixas de tráfego podem razoavelmente acomodar. Na interseção viária, as faixas de tráfego da AN foram as que mais tiveram sua capacidade utilizada, o que proporciona restrição de manobras, aumento do atraso médio por veículos e velocidade reduzida no tráfego de veículos.

A diferença entre os valores da relação v/c apresentados pelos softwares VISSIM e SimTraffic e o método Wesbster (1964) foram da ordem de centésimos. A divergência entre os níveis de serviço das faixas de tráfego determinados pelos softwares de microssimulação ocorreu apenas nos cenários 2 e 3, e quando houve divergência, a diferença foi entre níveis de serviço próximos. Tendo em vista tais observações, foram necessárias outras análises que investigassem a qualidade ou o nível de serviço em termos de medidas de desempenho operacional, tais como atraso médio por veículo e comprimento da fila de veículos nas faixas de tráfego.

A Figura 5 apresenta os resultados do atraso médio veicular em segundos (s) para os três cenários considerados, obtidos a partir das microssimulações e calculados conforme equação 5 pelo método de Webster (1964).

Figura 1. Comparação do parâmetro atraso médio por veículo.

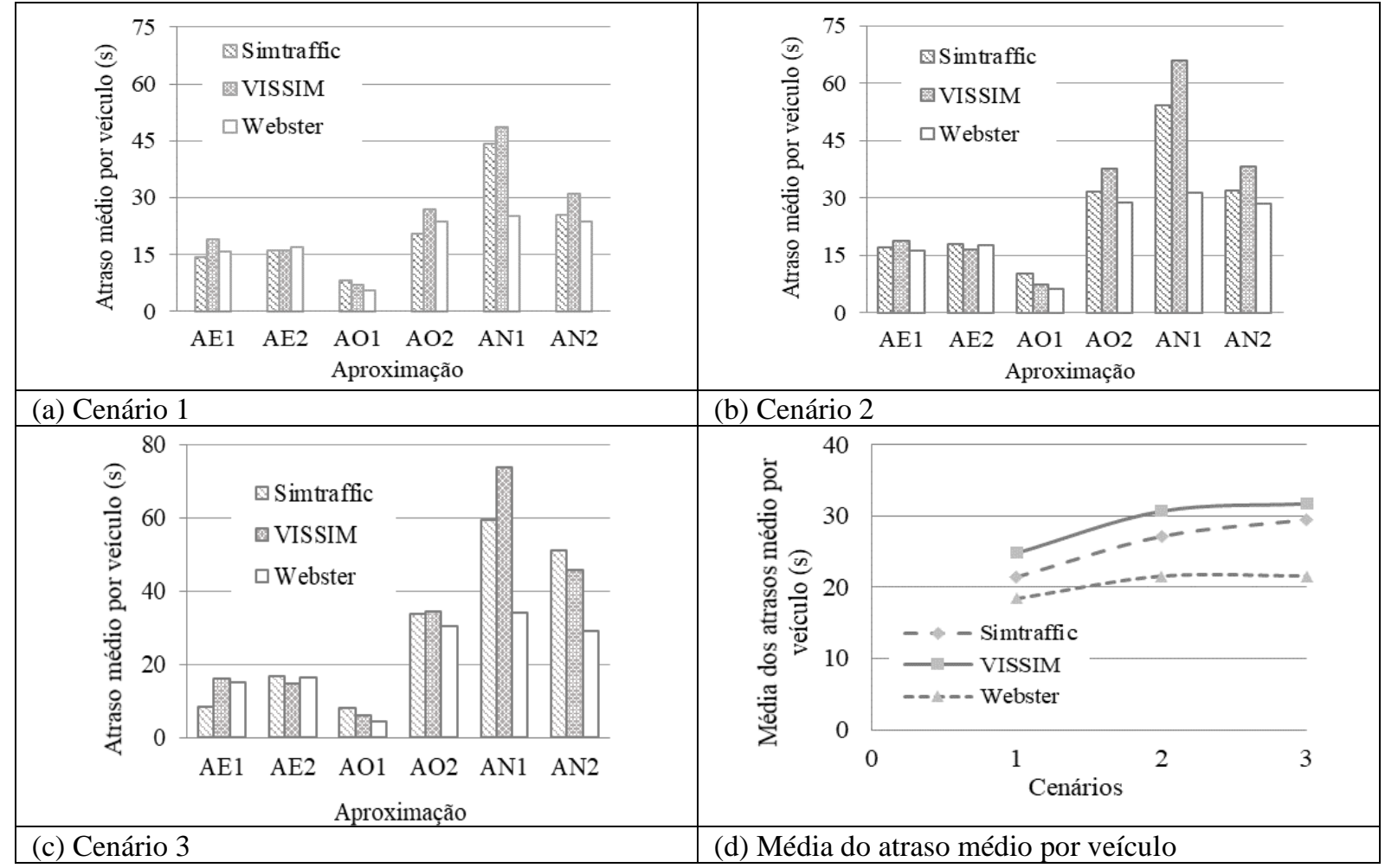

Fonte: Autores (2021).

Ao comparar os valores obtidos com as microssimulações para os dois softwares, observa-se que os atrasos ficaram próximos. A diferença máxima foi de 9 segundos, na aproximação AN1 do cenário C2, indicando que ambos os simuladores 
conseguiram reproduzir os mesmos cenários. Os atrasos médios (Figura 6d) obtidos pelo método de Webster (1964) e pelas microssimulações aumentam com o aumento do tempo de ciclo de 80 s para $95 \mathrm{~s}$.

Calculou-se o atraso pelo método de Webster (1964) para os volumes médios obtidos das 30 microssimulações de cada programa. A diferença entre o atraso calculado com o volume em campo e os volumes médios simulados com os dois softwares foi inferior a 1 segundo.

Os valores do comprimento da fila de veículos em metros (m) nas aproximações da interseção semaforizada, obtidos a partir das microssimulações e pelo método manual Webster (1964), para os três cenários, são apresentados na Figura 6.

Figura 6. Comparação do parâmetro comprimento da fila.

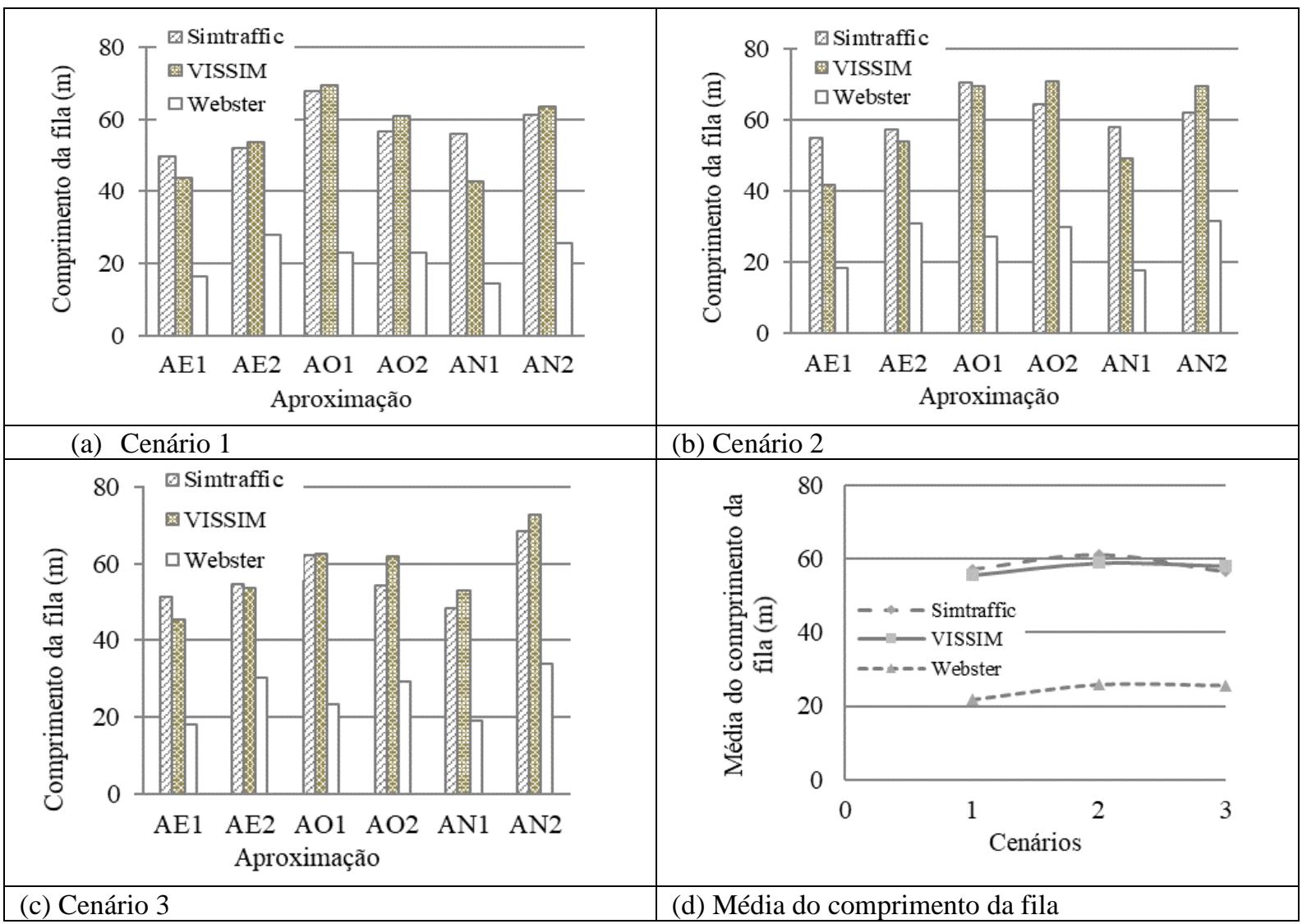

Fonte: Autores (2021).

Observa-se nos três cenários que os menores comprimentos da fila de veículos foram obtidos aplicando o método de Webster (1964), sendo estes valores inferiores à metade dos obtidos com as microssimulações. Para o cenário C1, o maior comprimento da fila foi obtido para a aproximação AO1, e para o C2 e C3 foi para a aproximação AN2.

Comparando os comprimentos da fila determinados pelos microssimulações, observa-se a proximidade entre os valores, cuja diferença máxima foi igual a 14 metros. Infere-se que a diferença entre o comprimento médio das filas de veículos determinados pelos microssimuladores, observados na Figura 3, pode estar relacionada com o comprimento dos veículos adotados pelos respectivos softwares, pois no SimTraffic o comprimento do veículo é igual a 5,94 metros e no VISSIM pode variar entre 3,75 e 4,76 metros. Chaudhry (2015) ainda considera que outro fator influenciador seja o método de determinação do comprimento médio da fila de veículos.

Os comprimentos da fila médios (Figura 6 d) obtidos pelo método de Webster (1964) (Equação 7) e microssimulações foram maiores no cenário correspondente ao ciclo ótimo calculado (C2). Para o método manual, os comprimentos de fila foram 
inferiores ao dobro dos valores obtidos com as microssimulações em ambos os softwares. A diferença entre o comprimento da fila calculado com o volume em campo e os volumes médios simulados foi inferior a 1metro.

\subsection{Análises estatísticas}

Para melhor compreender a qualidade ou o nível de serviço das faixas de tráfego apresentada pelos softwares microssimuladores utilizados nessa pesquisa, foram realizadas análises estatísticas dos parâmetros de desempenho operacional "atraso médio por veículo" e "comprimento da fila de veículos" nas faixas de tráfego, conforme apresentado nas Tabelas 4 e 5 , respectivamente.

Tabela 4. Análise estatística do atraso médio por veículo determinado pelos softwares VISSIM e SimTraffic.

\begin{tabular}{|c|c|c|c|c|c|c|c|c|c|}
\hline \multirow[b]{2}{*}{ Cenário } & \multirow[b]{2}{*}{ Aproximação } & \multirow{2}{*}{$\begin{array}{l}\text { Método } \\
\text { Webster } \\
\text { (1964) }\end{array}$} & \multicolumn{2}{|c|}{ VISSIM } & \multicolumn{2}{|c|}{ SimTraffic } & \multicolumn{3}{|c|}{$\begin{array}{c}\text { Análise estatística VISSIM x } \\
\text { SimTraffic } \\
\end{array}$} \\
\hline & & & Média (s) & $\begin{array}{c}\text { Desvio } \\
\text { padrão (s) }\end{array}$ & Média (s) & $\begin{array}{c}\text { Desvio } \\
\text { padrão } \\
\text { (s) }\end{array}$ & $\mathrm{u}$ & $\mathrm{F}$ & Valor-p \\
\hline \multirow{6}{*}{1} & AL1 & 15,76 & 19,07 & 3,91 & 14,38 & 1,88 & 95,0 & - & $<0,001 *$ \\
\hline & AL2 & 16,83 & 16,16 & 1,72 & 15,98 & 1,11 & - & 0,2 & 0,648 \\
\hline & $\mathrm{AO} 1$ & 5,45 & 6,97 & 1,21 & 8,20 & 0,94 & - & 19,6 & $<0,001 *$ \\
\hline & $\mathrm{AO} 2$ & 23,65 & 26,86 & 4,08 & 20,39 & 7,86 & 185,0 & - & $<0,001^{*}$ \\
\hline & AN1 & 25,19 & 48,64 & 12,51 & 44,21 & 7,77 & - & 2,717 & 0,105 \\
\hline & AN2 & 23,72 & 31,04 & 4,19 & 25,34 & 5,33 & 170,0 & - & $<0,001 *$ \\
\hline \multirow{6}{*}{2} & AL1 & 16,33 & 18,81 & 3,91 & 16,96 & 1,46 & 283,5 & - & $0,007^{*}$ \\
\hline & AL2 & 17,74 & 16,58 & 1,88 & 17,97 & 1,40 & - & 10,6 & $0,002 *$ \\
\hline & $\mathrm{AO} 1$ & 6,28 & 7,24 & 1,23 & 10,31 & 1,18 & - & 97,5 & $<0,001 *$ \\
\hline & $\mathrm{AO} 2$ & 28,92 & 39,64 & 9,43 & 31,44 & 1,74 & 126,0 & - & $<0,001 *$ \\
\hline & AN1 & 31,38 & 65,88 & 25,65 & 54,32 & 5,98 & 344,0 & - & 0,059 \\
\hline & AN2 & 28,53 & 38,28 & 3,73 & 31,79 & 9,09 & 184,5 & - & $<0,001 *$ \\
\hline \multirow{6}{*}{3} & AL1 & 14,95 & 16,01 & 3,44 & 8,51 & 1,51 & - & 120,1 & $<0,001 *$ \\
\hline & AL2 & 16,39 & 14,62 & 2,18 & 16,63 & 1,63 & - & 16,3 & $<0,001 *$ \\
\hline & $\mathrm{AO} 1$ & 4,42 & 6,06 & 1,66 & 8,07 & 0,88 & - & 34,3 & $<0,001 *$ \\
\hline & $\mathrm{AO} 2$ & 30,42 & 34,54 & 5,83 & 33,58 & 1,73 & - & 0,745 & 0,392 \\
\hline & AN1 & 33,94 & 73,78 & 33,41 & 59,51 & 15,54 & 360,0 & - & 0,092 \\
\hline & AN2 & 29,22 & 45,87 & 8,41 & 51,01 & 11,36 & 323,0 & - & $0,030^{*}$ \\
\hline
\end{tabular}

* Nível de significância de 5\%, valor-p $\leq 0.05$. F: Parâmetro do teste de Fisher proveniente do ANOVA, para dados paramétricos. u: Parâmetro do teste U de Mann-Whitney para dados não paramétricos. Fonte: Autores (2021).

Em relação aos valores de atraso médio por veículo determinados pelas simulações de tráfego em ambos os softwares, notou-se que a aproximação AN1 em comparação com as demais aproximações foi a que apresentou maiores valores de desvio padrão nos três cenários simulados. Além disso, destaca-se na aproximação AN1, em todos os cenários, a discrepância das médias do parâmetro atraso médio por veículos provenientes das microssimulações, em ambos os softwares, em relação aos valores desse parâmetro determinados pelo método Webster (1964). Isso ocorreu mesmo sendo a aproximação norte aquela com menor volume de tráfego.

É possível afirmar que essas observações estão relacionadas ao ponto de conflito, localizado a jusante do sistema de controle de tráfego, proporcionado pela conversão à esquerda permitida pelas AN1 e AN2, tendo como preferência os veículos 
provenientes da AN2, na qual existe uma redução de duas faixas (links) para uma. Em suma, não houve diferença significativa entre o atraso médio por veículo na aproximação AN1 determinado pelos softwares de microssimulação em nenhum dos cenários estudados e estes apresentaram discrepância com o calculado pelo método Webster (1964). Logo, inferiu-se que o método manual não representa o atraso real determinado em campo, em especial nos casos em que há pontos de conflitos com preferência de conversão entre as faixas de tráfego.

Além disso, observou-se que, com o aumento do ciclo semafórico, visto entre os cenários, o atraso na aproximação AL2 apresentou diferença significativa entre os softwares, mesmo com o aumento do tempo de verde.

Além das diferenças nos cálculos de atraso em cada modelo, os resultados da microssimulação podem ser específicos para as condições assumidas nos modelos. O comprimento do link e a velocidade de viagem são os dois elementos que não são considerados especificamente na metodologia HCM (2010). Suspeita-se que, quando um link está altamente saturado, os motoristas tendem a se comportar de maneira mais uniforme, resultando em variações reduzidas nas medidas de desempenho.

Comparando estatisticamente os atrasos médios obtidos com os microssimuladores, observa-se que, houve diferenças significativas em no mínimo três faixas por cenário dos três cenários analisados.

Tabela 5. Análise estatística do parâmetro comprimento da fila de veículos nas aproximações determinado pelos softwares.

\begin{tabular}{|c|c|c|c|c|c|c|c|c|c|}
\hline \multirow[b]{2}{*}{ Cenário } & \multirow[b]{2}{*}{ Aproximação } & \multirow{2}{*}{$\begin{array}{c}\text { Método } \\
\text { Webster } \\
(1964)\end{array}$} & \multicolumn{2}{|c|}{ VISSIM } & \multicolumn{2}{|c|}{ Simtraffic } & \multicolumn{3}{|c|}{$\begin{array}{c}\text { Análise estatística VISSIM x } \\
\text { SimTraffic } \\
\end{array}$} \\
\hline & & & $\begin{array}{l}\text { Média } \\
(\mathrm{m})\end{array}$ & $\begin{array}{l}\text { Desvio } \\
\text { padrão } \\
(\mathrm{m})\end{array}$ & $\begin{array}{l}\text { Média } \\
(\mathrm{m})\end{array}$ & $\begin{array}{l}\text { Desvio } \\
\text { padrão } \\
(\mathrm{m})\end{array}$ & $\mathrm{u}$ & $\mathrm{F}$ & Valor-p \\
\hline \multirow{6}{*}{1} & AL1 & 16,52 & 43,62 & 12,25 & 49,82 & 6,56 & - & 5,6 & $0,017^{*}$ \\
\hline & AL2 & 27,87 & 53,62 & 3,56 & 51,87 & 5,78 & 378,0 & - & 0,14 \\
\hline & $\mathrm{AO} 1$ & 23,13 & 69,57 & 23,72 & 67,78 & 13,41 & 426,0 & - & 0,36 \\
\hline & $\mathrm{AO} 2$ & 23,17 & 60,91 & 19,44 & 56,64 & 13,11 & 427,5 & - & 0,37 \\
\hline & AN1 & 14,38 & 42,78 & 17,25 & 55,82 & 7,94 & - & 14,2 & $<0,001 *$ \\
\hline & AN2 & 25,66 & 63,45 & 12,17 & 61,11 & 6,01 & 428,0 & - & 0,37 \\
\hline \multirow{6}{*}{2} & AL1 & 18,27 & 41,54 & 11,39 & 54,81 & 10,06 & 174,0 & - & $<0,001 *$ \\
\hline & AL2 & 30,84 & 53,70 & 4,30 & 57,12 & 6,51 & 296,5 & - & $0,012^{*}$ \\
\hline & AO1 & 26,99 & 69,39 & 23,32 & 70,42 & 10,34 & - & 0,05 & 0,826 \\
\hline & $\mathrm{AO} 2$ & 29,73 & 70,69 & 24,73 & 64,40 & 13,43 & 389,0 & - & 0,184 \\
\hline & AN1 & 17,63 & 48,99 & 20,06 & 58,01 & 7,14 & 308,0 & - & $0,018^{*}$ \\
\hline & AN2 & 31,46 & 69,43 & 13,76 & 62,10 & 4,75 & 278,0 & - & $0,006^{*}$ \\
\hline \multirow{6}{*}{3} & AL1 & 17,92 & 45,22 & 10,90 & 51,45 & 7,56 & 311,5 & - & $0,02 *$ \\
\hline & AL2 & 30,25 & 53,61 & 4,15 & 54,46 & 6,45 & 393,0 & - & 0,20 \\
\hline & AO1 & 23,13 & 62,39 & 24,35 & 62,29 & 10,66 & - & 0,01 & 0,98 \\
\hline & $\mathrm{AO} 2$ & 29,34 & 63,02 & 16,57 & 54,25 & 10,95 & 295,0 & - & $0,01^{*}$ \\
\hline & AN1 & 19,02 & 52,92 & 19,85 & 48,27 & 7,29 & 432,5 & - & 0,40 \\
\hline & AN2 & 33,94 & 72,87 & 8,37 & 68,45 & 3,75 & 243,0 & - & $0,00 *$ \\
\hline
\end{tabular}

* Nível de significância de 5\%, valor-p $\leq 0.05$. Fonte: Autores (2021).

Como observado na Tabela 5, em aproximadamente 50\% das análises estatísticas realizadas, verificou-se diferença significativa entre o comprimento da fila de veículos nas aproximações da interseção viária determinado pelos softwares VISSIM e SimTraffic. Nas análises, $78 \%$ dos maiores valores de comprimento da fila de veículos foram provenientes das microssimulações realizadas no software SimTraffic. Esse fato pode estar relacionado ao método de determinação do 
comprimento médio das filas de veículos e com o comprimento dos veículos considerados em cada software de microssimulação.

Além disso, destaca-se a discrepância entre os valores do comprimento das filas dos veículos derivados das simulações de tráfego e aqueles determinados pelo método manual de Webster (1964). Esta diferença pode ser atribuída ao fato do método manual não considerar a distância entre os veículos, a existência de diferentes tipos de condutores, a variação das velocidades ou a quantidade e tipos de movimentos existentes.

\section{Considerações Finais}

Os parâmetros de desempenho operacional, atraso médio por veículo e comprimento da fila de veículos nas faixas de tráfego podem ser utilizados na análise da influência de fatores no desempenho operacional de interseções. Os valores dos parâmetros de desempenho operacional apresentados pelos modelos de simulações dos softwares VISSIM e Simtraffic retratam melhor a realidade do tráfego na interseção viária em estudo do que aqueles parâmetros obtidos pela aplicação do método manual de Webster (1964). Entretanto, cabe destacar que a relação volume de tráfego e capacidade obtidos pelos softwares VISSIM e Simtraffic, e o método Webster (1964) apresentaram valores aproximados.

Tendo em vista os diferentes cenários e aproximações estudadas, a análise estatística dos parâmetros "atraso médio por veículo" e "comprimento médio da fila" determinados pelas simulações de tráfego nos softwares Vissim e Simtraffic, conclui-se que no parâmetro "atraso médio por veículo" a recorrência de diferença estatística significativa entre os valores apresentados pelos softwares foi maior do que no parâmetro "comprimento médio da fila". Por isso, infere-se que não são todos os parâmetros de desempenho operacional de uma interseção viária que variam conforme o software simulador do tráfego e essa variação depende dos diferentes fatores existentes nos cenários de tráfego simulado.

Desse modo, nota-se a importância da aplicação de testes estatísticos, como o ANOVA e Mann-Whitney, no planejamento do tráfego urbano, haja vista que tais testes determinam se os parâmetros desempenho operacional e quais destes se diferenciam conforme o cenário, métodos e softwares aplicados. Estas informações auxiliam os planejadores na tomada de decisão, quanto as alterações e melhorias nas interseções semafóricas, pois a escolha do método e software através da análise dos parâmetros de desempenho operacional permite uma simulação de cenários alternativos mais próximos das situações existentes em campo.

Destaca-se que os valores do parâmetro "comprimento médio da fila", determinado pelo método Webster (1964) foram muito inferiores em relação aqueles determinados pelos modelos de simulação de tráfego, mesmo naquelas aproximações em que os valores dos parâmetros determinados pelos modelos de simulação apresentaram diferença estatística significativa.

Diante do exposto recomenda-se que pesquisas futuras comparem, a partir de testes estatísticos, outros parâmetros de eficiência de interseções semafóricas, por exemplo, o nível de serviço ou a capacidade das aproximações, e considere diferentes cenários e softwares de simulação de tráfego.

\section{Referências}

Araújo, A. M., \& Castro, M. M. (2018). Calibração do modelo de aceitação de brechas em interseções urbanas com o microssimulador de tráfego VISSIM. Transportes, 26(3), 25-38. 10.14295/transportes.v26i3.1603

ATAC. (2003). Signal coordination strategies Final Report. Grand Forks/East grand forks metropolitan planning. Fargo, North Dakota.

Ayala, R. J. L. E (2013). Procedimento para identificação dos principais parâmetros dos microssimuladores a serem considerados no processo de calibração. Dissertação de mestrado, Universidade de Brasília, Faculdade de Tecnologia, Brasília.

Bloomberg, L., \& Dale, J. (2000). Comparison of VISSIM and CORSIM traffic simulation models on a congested network. Transportation Research Record, (1727), 52-60. 10.3141/1727-07 
Cal, R., Reyes, M., \& Cárdenas, J. (2010). Ingeniería de Tránsito fundamentos y aplicaciones. Félix Varela, Ed 8va. México.

Chaudhry, A. (2015). Analysis of queue characteristics at signalized intersections near highway-railroad grade crossing. Dissertation, Wayne State University, Detroit, Michigan.

Chaudhry, M. S. \& Ranjitkar, P. (2013). Delay Estimation at Signalized Intersections with Variable Queue Discharge Rate. Journal of the Eastern Asia Society for Transportation Studies, 10(0).

Datta, S., Rokade, S., \& Rajput, S. P. S. (2020). Delay and driver turning time evaluation for uncontrolled intersections under diverse traffic operational situations. Transportation Engineering, 2(May), 100031. 10.1016/j.treng.2020.100031

De Oliveira, S. (2016) Calibração de modelos de microssimulação de tráfego para análise de interseções urbanas. Dissertação de mestrado, Instituto Politécnico de Viseu.

Departamento Nacional de Infraestrutura de Transportes. Manual de estudos de tráfego. Instituto de Pesquisas Rodoviárias - IPR 384 (2006). Rio de Janeiro.

Dion, F., Rakha, H., \& Kang, Y. S. (2004). Comparison of delay estimates at under-saturated and over-saturated pre-timed signalized intersections. Transportation Research Part B: Methodological, 38(2), 99-122. https://doi.org/10.1016/S0191-2615(03)00003-1.

Dowling, R., Skabardonis, A., \& Alexiadis, V. (2004). Traffic Analysis Toolbox Volume III : Guidelines for Applying Traffic Microsimulation Modeling Software. Rep. No. FHWA-HRT-04-040, U.S. DOT, Federal Highway Administration, Washington, D.C (Vol. III).

Elefteriadou, L. (2014). An Introduction to Traffic Flow Theory (Springer). Vol. 84.

Jíménez, J. A., Medina, J. M., Figueroa, V., \& Tapia, M. (2015). Simulación con Promodel para la sincronización de semáforos. Academia Journals, 1, 11871192 .

Khosla, K., \& J. C. Williams (2006). Saturation flow at signalized intersections during longer green time. Transportation Research Record: Journal of the Transportation Research Board, 1978, 61-67. 10.3141/1978-10.

Koonce, P., Rodegerdts, L., Lee, K., Quayle, S., Beaird, S., Braud, C., Bonneson, J., Tarnoff, P., \& Urbanik, T. (2008) Traffic Signal Timimg Manual (FHWAHOP-08-024). Department of Transportation, Federal Highway Administration

Kumar, R., \& Dhinakaran, G. (2013). Estimation of delay at signalized intersections for mixed traffic conditions of a developing country. International Journal of Civil Engineering, 11(1), 53-59.

Lee, M. (2010). Performance Analysis of the Dowling Multi-lane Roundabouts in Anchorage, Alaska University Transportation Center. Alaska Department of Transportation and Public Facilities, Alaska.

Ma, W., Wan, L., Yu, C., Zou, L., \& Zheng, J. (2020). Multi-objective optimization of traffic signals based on vehicle trajectory data at isolated intersections. Transportation Research Part C: Emerging Technologies, 120(September), 102821. 10.1016/j.trc.2020.102821

Mahmud, S. M. S., Ferreira, L., Hoque, M. S., \& Tavassoli, A. (2019) Micro-simulation modelling for traffic safety: A review and potential application to heterogeneous traffic environment. IATSS Research, 43(1), 27-36. 10.1016/j.iatssr.2018.07.002

Nyame-Baafi, E., Adams, C. A., \& Osei, K. K. (2018). Volume warrants for major and minor roads left-turning traffic lanes at unsignalized T-intersections: A case study using VISSIM modelling. Journal of Traffic and Transportation Engineering (English Edition), 5(5), 417-428. 10.1016/j.jtte.2018.01.005

Obelheiro, M. R., Oliveira, M. L., \& Cybis, H. B. B. (2010). Avaliação da influência do tipo de cobrança e da composição do tráfego sobre o desempenho operacional de praças de pedágio. Transportes, 18(3), 25-33. 10.14295/transportes.v18i3.448

Oskarbski, J., Guminska, L., Miszewski, M., \& Oskarbska, I. (2016). Analysis of Signalized Intersections in the Context of Pedestrian Traffic. Transp. Res. Procedia, 14, 2138-2147. 10.1016/j.trpro.2016.05.229.

Osorio, C., Chen, X., Marsico, M., Talas, M., Gao, J., \& Zhang, S. (2015). Reducing Gridlock Probabilities via Simulation-based Signal Control. Transportation Research Procedia, 6(June 2014), 101-110. 10.1016/j.trpro.2015.03.009

Otković, I. I., Deluka-Tibljaš, A., \& Šurdonja, S. (2020). Validation of the calibration methodology of the micro-simulation traffic model. Transportation Research Procedia, 45(2019), 684-691. 10.1016/j.trpro.2020.02.110

Park, B., \& Qi, H. (2005). Development and evaluation of a procedure for the calibration of simulation models. Transportation Research Record, (1934), 208217. $10.3141 / 1934-22$.

PTV AG Corporation. (2016). Vissim 9 user manual. Karlsruhe, Germany.

Raval, N. G., \& Gundaliya, P. J. (2012). Modification of Webster's delay formula using modified saturation flow model for non-lane based heterogeneous traffic condition. Highway Research Journal, 5(1).

Saha, A., Chandra, S., \& Ghosh, I. (2016). A Comparison of Delay at Signal Controlled Intersections Based on Different Methods. 12th Transportation Planning and Implementation Methodologies for Developing, (March 2017).

Shaaban, K. (2005). Right Turn Split: A New Design to Alleviate the Weaving Problem on Arterial Streets. Electronic Theses and Dissertations, $2004-2019$. Department of Civil and Environmental Engineering. College of Engineering and Computer Science. University of Central Florida.

Shaaban, K., \& Kim, I. (2015). Comparison of SimTraffic and VISSIM microscopic traffic simulation tools in modeling roundabouts. Procedia Computer Science, 52(1), 43-50. 10.1016/j.procs.2015.05.016 
Research, Society and Development, v. 11, n. 3, e0511326178, 2022

(CC BY 4.0) | ISSN 2525-3409 | DOI: http://dx.doi.org/10.33448/rsd-v11i3.26178

Shaaban, K., \& Radwan, E. (2005). A Calibration and Validation Procedure for Microscopic Simulation Model : A Case Study of SimTraffic for Arterial Streets. TRB (Ed), TRB 2005 Annual Meeting. Transportation Research Board, National Research Council, Washington, Orlando.

Steven L. Jones, Andrew J. Sullivan, Cheekoti, N., Michael D. Anderson, \& Dillip Malave. (2004). Traffic simulation software comparison study. UTCA Report 02217 (Vol. 2217). Alabama. 10.1.1.117.6974

Sun, D., Zhang, L., \& Chen, F. (2013). Comparative study on simulation performances of CORSIM and VISSIM for urban street network. Simulation Modelling Practice and Theory, 37, 18-29. 10.1016/j.simpat.2013.05.007

Teixeira, C. A. (2008). Calibração de Modelos Microscópicos de Simulação de Tráfego em Redes Urbanas Cristina. Dissertação de mestrado. Faculdade de engenharia. Universidade do Porto.

Tianzi, C., Shaochen, J., \& Hongxu, Y. (2013). Comparative Study of VISSIM and SIDRA on Signalized Intersection. Social and Behavioral Sciences, 96(Cictp), 2004-2010. 10.1016/j.sbspro.2013.08.226

Torquato, R. M., Maciel, M., \& de Castro Neto, M. (2020). Estimação do efeito de motocicletas na capacidade de interseções semaforizadas. Transportes, $28(5), 220-234$.

Trafficware, L. (2017). Synchro Studio 10: Synchro plus SimTraffic and 3D Viewer. Trafficware, LLC. 522 Gillingham Sugar Land, TX 77487, United States of America.

TRB (2010). HCM 2010: Highway Capacity Manual. 5th Edition. Transportation Research Board - National Research Council. Washington, D.C.

Wang, C., Xu, C., Xia, J., Qian, Z., \& Lu, L. (2018). A combined use of microscopic traffic simulation and extreme value methods for traffic safety evaluation. Transportation Research Part C: Emerging Technologies, 90 (December 2017), 281-291. 10.1016/j.trc.2018.03.011

Wang, Z., Zhu, L., Ran, B., \& Jiang, H. (2020). Queue profile estimation at a signalized intersection by exploiting the spatiotemporal propagation of shockwaves. Transportation Research Part B: Methodological, 141, 59-71. 10.1016/j.trb.2020.08.009

Webster, F. (1964). Traffic signal settings, road research technical paper no. 39”. Road Research Laboratory. Londres.

Yizheng, W., Jian, S., \& Chunlu, P. (2012). Comparative Study of Micro-simulation Performances for Urban Road Network between Vissim and Corsim. Traffic Information and Safety, 30(4), 116-120. 